\title{
Development of a New Monomer for the Synthesis of Intrinsic Antimicrobial Polymers with Enhanced Material Properties
}

\author{
Florian Brodkorb ${ }^{1, \dagger}, *$, Björn Fischer ${ }^{1, \dagger}$, Katrin Kalbfleisch ${ }^{1, \dagger}$, Oliver Robers ${ }^{1, \dagger}$, Carina Braun ${ }^{2}$, \\ Sophia Dohlen ${ }^{2}$, Judith Kreyenschmidt ${ }^{2}$, Reinhard Lorenz ${ }^{1}$ and Martin Kreyenschmidt ${ }^{1}$ \\ 1 Institute of Construction and Functional Materials, University of Applied Sciences Münster, \\ Stegerwaldstraße 39, 48565 Steinfurt, Germany; E-Mails: bjoern.fischer@fh-muenster.de (B.F.); \\ katrin.kalbfleisch@fh-muenster.de (K.K.); o.robers@fh-muenster.de (O.R.); \\ rlorenz@fh-muenster.de (R.L.); martin.kreyenschmidt@fh-muenster.de (M.K.) \\ 2 Institute of Animal Science, University Bonn, Katzenburgweg 7-9, 53113 Bonn, Germany; \\ E-Mails: cbraun@uni-bonn.de (C.B.); sdohlen@uni-bonn.de (S.D.); \\ j.kreyenschmidt@uni-bonn.de (J.K.) \\ $\dagger$ These authors contributed equally to this work. \\ * Author to whom correspondence should be addressed; E-Mail: f.brodkorb@fh-muenster.de; \\ Tel.: +49-255-1962-559; Fax: +49-255-1962-429.
}

Academic Editor: Antonella Piozzi

Received: 25 June 2015 / Accepted: 17 August 2015 / Published: 24 August 2015

\begin{abstract}
The use of biocidal compounds in polymers is steadily increasing because it is one solution to the need for safety and hygiene. It is possible to incorporate an antimicrobial moiety to a polymer. These polymers are referred to as intrinsic antimicrobial. The biocidal action results from contact of the polymer to the microorganisms, with no release of active molecules. This is particularly important in critical fields like food technology, medicine and ventilation technology, where migration or leaching is crucial and undesirable. The isomers $N$-(1,1-dimethylethyl)-4-ethenyl-benzenamine and $N$-(1,1-dimethyl-ethyl)-3ethenyl-benzenamine (TBAMS) are novel (Co-)Monomers for intrinsic anti-microbial polymers. The secondary amines were prepared and polymerized to the corresponding water insoluble polymer. The antimicrobial activity was analyzed by the test method JIS Z 2801:2000. Investigations revealed a high antimicrobial activity against Staphylococcus aureus and Escherichia coli with a reduction level of $>4.5 \log _{10}$ units. Furthermore, scanning electron
\end{abstract}


microscopy (SEM) of E. coli. in contact with the polymer indicates a bactericidal action which is caused by disruption of the bacteria cell membranes, leading to lysis of the cells.

Keywords: monomer; antimicrobial polymer; biocide; intrinsic antimicrobial

\section{Introduction}

The use of biocidal compounds in polymers is steadily increasing because it is one solution to the need for safety and hygiene. A compound that kills or inhibits the growth of microorganisms is referred to as a biocide. Potential fields of application include for example hospital surfaces/furniture, surgery equipment, health care products or food packaging. Two different categories of antimicrobial materials are differentiated. The biocide can be either temporarily trapped in the polymeric matrix or permanently attached to the backbone of the polymer. Commonly, a low molecular weight biocide is released from a plastic material, in which it is physically entrapped. The 2,4,4'-trichoro-2-hydroxydiphenyl (Triclosan) is the most frequently biocide applied in this strategy [1-3]. Further agents used are derivatives of isothiazolone, chlorine releasing $N$-halamines [4], nanosilver [5,6], as well as salts and complexes of metals (typically $\mathrm{Cu}$ and $\mathrm{Zn}$ ) $[7,8]$.

It is also possible to incorporate an antimicrobial moiety to the polymer; these polymers are referred to as intrinsic antimicrobial as the biocidal action results from contact of the polymer and the microorganisms, with no release of the active molecule $[9,10]$. Typical examples are polymers substituted by quaternary ammonium salts [11-16], phosphonium salts [17-20], and pyridinium groups [21-23], respectively.

Poly[2-(tert-butylamino)ethyl methacrylate] (poly(TBAEMA)) is a typical representative of a water-insoluble biocide, its structure is displayed in Figure 1. It was developed in 2001 by Creavis Technologies and Innovation of the Degussa (Marl, Germany). This polymer class was referred as sustainable active microbicidal (SAM-Polymers ${ }^{\circledR}$, Marl, Germany). According to the patent literature, poly(TBAEMA) exhibits inherent biocidal properties and has shown potential application in antifouling paints and coatings [24-27] or denture base acrylic resins [28,29]. The antimicrobial activity is caused by the pendant bulky secondary amine of the methacrylate backbone, without the need for quaternization, as is the case for other amine-containing polymers [30]. It must be noted that, although poly(TBAEMA) has high antimicrobial activity and low toxicity, the parent monomer shows no antimicrobial effect even at higher concentrations. This finding suggests that the antimicrobial action is closely related to the molecular structure and conformation of the polymer chains [30]. It has been reported that the solubility of poly(TBAEMA) in water (at $\mathrm{pH}=7$ ) is lower than $3.0 \mathrm{mg} / \mathrm{L}$. This renders the biocide especially useful for construction materials designed to be in contact with water, since a very low leachability of poly(TBAEMA) from polymer blends and compounds can be expected [31]. A major drawback of poly(TBAEMA) is the low glass transition temperature $\left(T_{\mathrm{G}}\right)$ of $40^{\circ} \mathrm{C}$ which limits the application as the surface becomes sticky at elevated temperatures. Other weak points are: high water uptake and a tendency for hydrolysis which yields 2-tert-butylamino-ethanol. The aim of this study was the development of a new monomer for this class of $\mathrm{SAM}^{\circledR}$-polymers, especially raising the $T_{\mathrm{G}}$ reducing water uptake and avoiding hydrolysis [32]. 


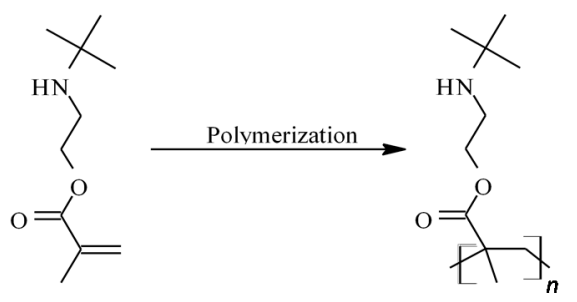

Figure 1. Structure of 2-(tert-butylamino) ethyl methacrylate (TBAEMA) and its resulting polymer poly-[2-(tert-butylamino) ethyl methacrylate] (poly(TBAEMA)).

\section{Results and Discussion}

\subsection{Synthesis of Aminomtehylstyrenes}

In order to screen a wider variety of different polymers for their glass transition temperatures, a series of aminomethylstyrenes was synthetized using a mixture of meta- and para-Isomers of vinylbenzyl chloride (VBC) and primary amines as starting materials (Figure 2). The reaction proceeds via an $\mathrm{S}_{\mathrm{N}} 2$ mechanism. In order to eliminate salt formation of the secondary amine an excess of sodium hydroxide $(\mathrm{NaOH})$ was used. For a high conversion and short reaction times a twofold excess of amine was applied. The reaction can be carried out under mild conditions due to the high reactivity of the benzyl chloride moiety. Success of the reaction was confirmed by GC-MS and Infrared spectroscopy (IR). The yields were moderate to good $(65 \%-80 \%)$, depending on the amine used. The structures of the alkylaminomethylstyrenes are displayed in Figure 3.

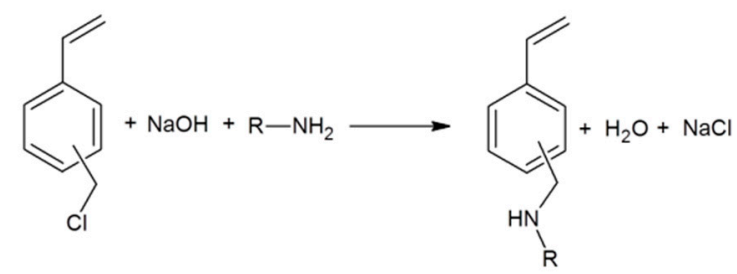

Figure 2. Reaction scheme aminomethylstyrene synthesis.

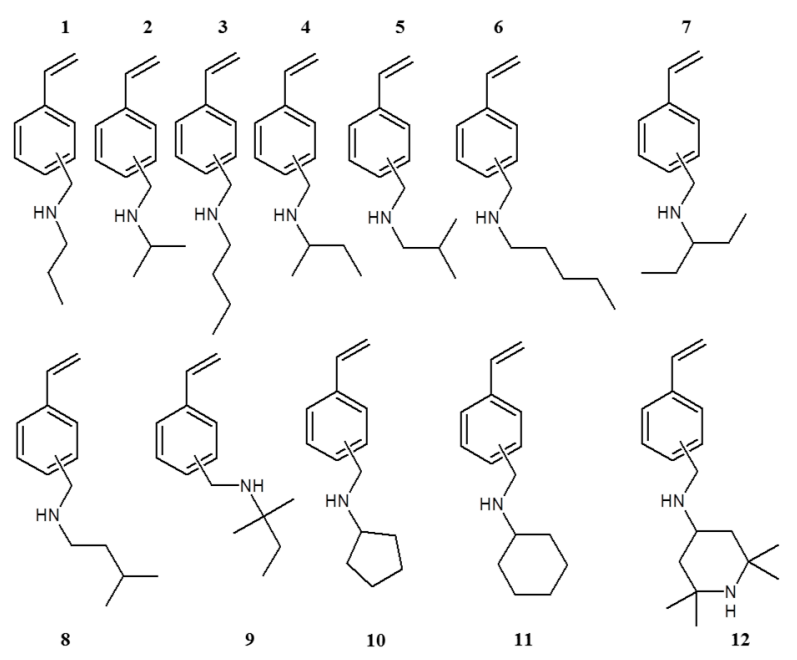

Figure 3. Structures of the alkylaminomethylstyrenes used for screening purpose. 
The monomers were polymerized as described in Section 3.1. Success of the polymerization was confirmed by IR, where the vinyl bands (e.g., 1630, 988, $903 \mathrm{~cm}^{-1}$ ) vanished after polymerization of the mlkylaminomethylstyrenes. The polymers synthetized from monomers 1-6, 8 and 12 showed a pronounced solubility in water, and were very soft and sticky. In contrast the polymers resulting from the polymerization of the monomers $\mathbf{7}$ and 9-11, were insoluble in water and isopropyl alcohol $(5 \% v / v)$. Additionally, they were more rigid and less sticky than the others. Determination of the glass transition temperature of the polymers formed by polymerization of the monomers 1-12 was not possible within the range of $25-160{ }^{\circ} \mathrm{C}$, as no melting peak was observed. Most likely $T_{\mathrm{G}}$ is below room temperature. The polymers of the monomers 1-12 were not suited for technical application because of the softness and stickiness in combination with a $T_{\mathrm{G}}$ below room temperature and the water solubility of some of them. The poly (tert-pentylaminomethylstyrole) (prepared from 9) had the best characteristics observed in this study so far. Thus, the tert-butyl derivative (poly(TBAMS)) may be a candidate that has the desired properties.

\subsection{Synthesis of N-(1,1-Dimethylethyl)-ethenyl-benzenamine (TBAMS)}

The general procedure for the synthesis of the alkylaminomethylstyrenes was optimized for higher yields employing a 3-fold excess of tert-butalymine and increasing the reaction temperature to $70{ }^{\circ} \mathrm{C}$ (Figure 4). Success of the reaction was confirmed by GC-MS, APCI-MS, IR and NMR. The GC-MS showed two new peaks corresponding to the meta- and para-isomers of TBAMS (57\% meta, 43\% para), whereas the VBC signals, resulting from the starting material, were very small (3\% of the total area in GC-MS). The EI-MS of the product peaks exhibit the molecular ion at $189 \mathrm{~m} / \mathrm{z}$. The signal at $174 \mathrm{~m} / \mathrm{z}$ be rationalized a methyl loss, whereas the $117 \mathrm{~m} / \mathrm{z}$ can be interpreted as a 4-(ethenyl phenyl)methylium ion that was formed by the loss of the tert-butyl amine moiety from the parent ion. The mass peak at $57 \mathrm{~m} / \mathrm{z}$ corresponds to the tert-butyl group. The infrared spectrum showed at $3306 \mathrm{~cm}^{-1}$ a weak band that can be addressed as the $v(\mathrm{NH})$ vibration. In the $\mathrm{APCI}^{+}$mass spectrum the protonated pseudo molecular ion at $m / z 190[\mathrm{M}+\mathrm{H}]^{+}$was observed. The major fragments are at $m / z 174$ represents the $\left(\mathrm{M}-\mathrm{CH}_{4}\right)^{+}$, $\mathrm{m} / \mathrm{z} 132$ the (M-tert-butyl) ${ }^{+}$(only observed after collision fragmentation of the isolated $\mathrm{m} / \mathrm{z} 190$ ) and $\mathrm{m} / \mathrm{z}$ 117 for the loss of tert-butylamin. The structure was further confirmed by ${ }^{1} \mathrm{H}$ and ${ }^{13} \mathrm{C}$ NMR (Figure 5).

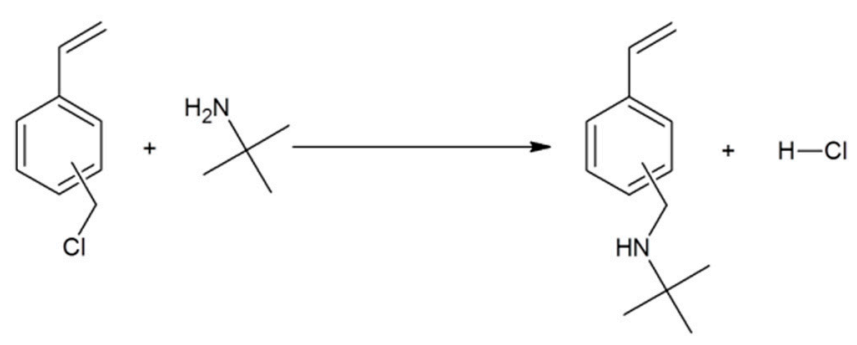

Figure 4. Reaction scheme of 2-(tert-butylamino) methylstyrene (TBAMS) synthesis. 
(A)

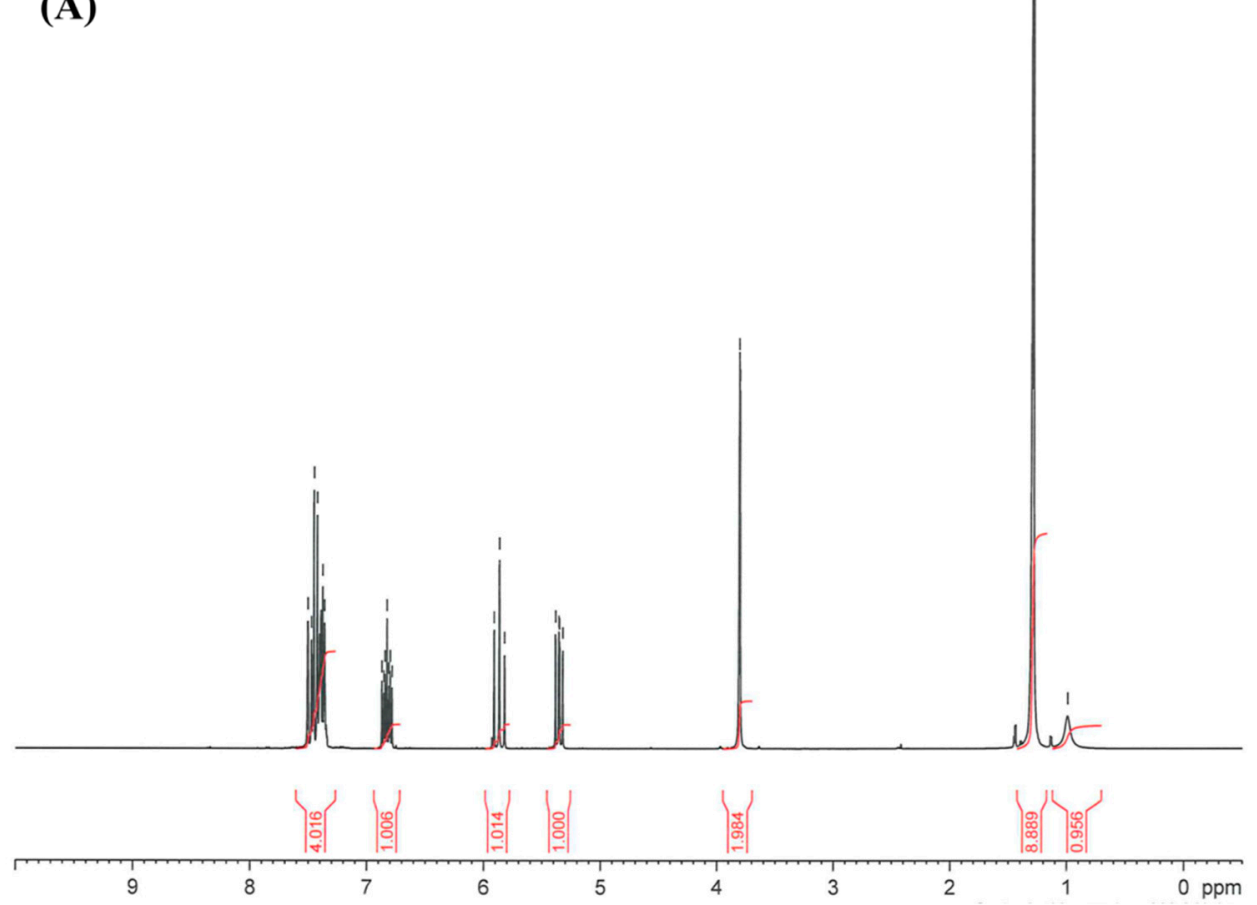

(B)

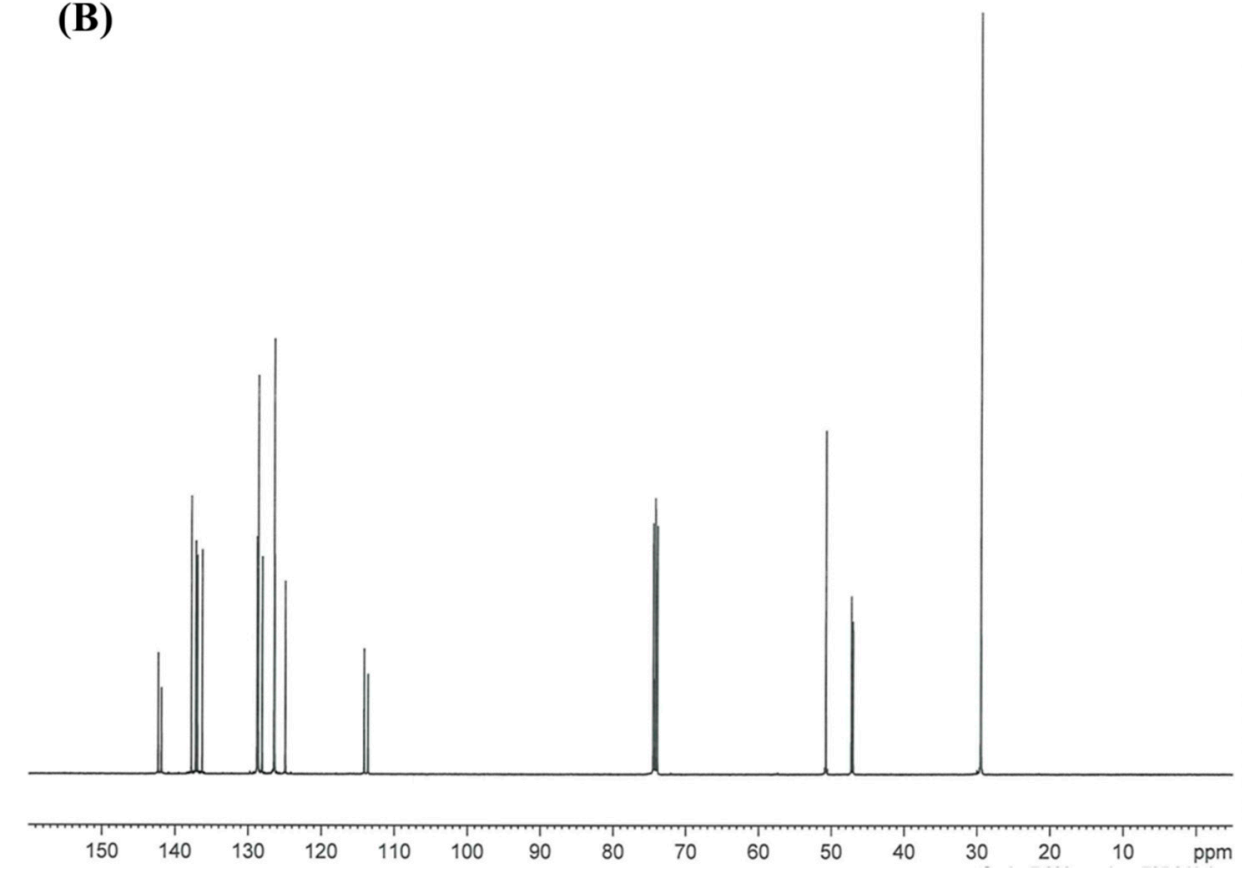

Figure 5. (A) ${ }^{1} \mathrm{H}$ NMR of TBAMS; (B) ${ }^{13} \mathrm{C}$ NMR of TBAMS.

\subsection{Synthesis of TBAMS Hydrochloride}

For elemental analysis and further characterization of TBAMS the hydrochloride was prepared. The elemental analysis showed a good match of calculated $\mathrm{C} / \mathrm{N} / \mathrm{H}$-ratios and experimentally determined values. The IR spectrum contains structural features (e.g., the bands at 2742, 2667, $2623 \mathrm{~cm}^{-1}$, etc.) that can be associated to the ammonium group in the hydrochloride. If the TBAMS hydrochloride is analyzed by GC-MS, the salt decomposes to $\mathrm{HCl}$ and TBAMS at the high temperature of the injector. Accordingly TBAMS was observed in the GC-MS chromatogram instead of TBAMS hydrochloride. As mentioned 
in Section 2.1 traces of VBC were present even in the distilled TBAMS, because the difference of the boiling points of VBC and TBAMS is too small to allow a quantitative separation in a single distillation step. As no residual VBC was present in the GC-MS analysis of TBAMS hydrochloride, the hydrochloride preparation offers a facile way to purify TBAMS.

\subsection{Preparation of Poly(TBAMS)}

Success of the polymerization was confirmed by ${ }^{1} \mathrm{H}$ and ${ }^{13} \mathrm{C}$ NMR (Figure 7). First experiments to polymerize TBAMS in bulk resulted in small molecular weight polymers. Therefore it was necessary to increase the molecular weight to an acceptable level for technical application. Accordingly polymerization of TBAMS was carried out in ethanol using 2,2'-azobisisobutyronitrile (AIBN) as initiator(Figure 6). The molecular weight $(M \mathrm{w})$ was raised to about $185 \mathrm{kDa}$ in typical experiments. The polydispersity $(M \mathrm{w} / M \mathrm{n})$ of 5.2 is rather high. A pronounced polydispersity (2.5-3) is typical for AIBN initiated radical reactions. The high polydispersity observed in the poly(TBAMS) polymer can be an indication, that grafting occurs during polymerization. Probably chain transfer occurs at the benzylic position of poly(TBAMS) and TBAMS. Poly(TBAMS) possesses a $T_{\mathrm{G}}$ of about $68^{\circ} \mathrm{C}$, which is considerably higher than the $T_{\mathrm{G}}$ of poly(TBAEMA). The poly(TBAMS) was soluble in ethanol and formed uniform, colorless and transparent films upon solvent evaporation. Glass transition temperatures can be further be adjusted by copolymerization to about $160^{\circ} \mathrm{C}$ without the loss of the antimicrobial activity. The copolymer was synthetized using $42 \mathrm{~mol} \%$ TBAMS, $42 \mathrm{~mol} \%$ 4-vinylpyridine and $16 \mathrm{~mol} \%$ 4-vinylbenzoic acid. The $\log$ reduction of this copolymer was 3.5 for $S$. aureus.

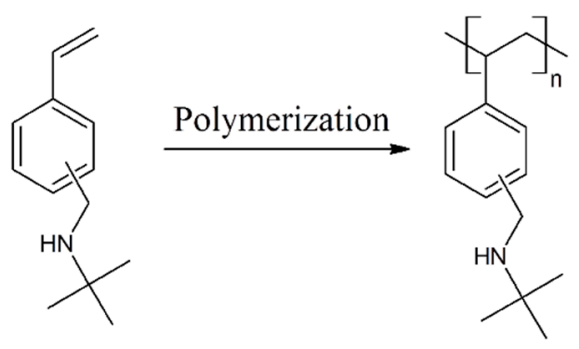

Figure 6. Structure of 2-(tert-butylamino) methylstyrene (TBAMS) and its resulting polymer.

Chitosan is a well-studied intrinsic antimicrobial polymer, which is a possible candidate for technical application. The glass transition temperature varies over a broad range between 61 and $203{ }^{\circ} \mathrm{C}$, depending on molecular weight, degree of deacetylation and residual water content [33-35]. A drawback of chitosan is the wet processing of the polymer to form films or blends with other polymers. Depending on the mode of preparation, a high water uptake and swelling in aqueous media is observed. The poly(TBAMS) has a lower water uptake of approximately 3\%. Another intrinsic antimicrobial polymer with a $T_{\mathrm{G}}$ of $218^{\circ} \mathrm{C}$ is a poly(styrene-alt-maleic anhydride)-4-aminophenol conjugate [36]. The antimicrobial properties of this polymer regarding the reduction of E. coli and $S$. aureus are good but cannot be directly compared to poly(TBAMS) as different methods were used to determine the reduction rates. Methylacrylamide based polymers and copolymers described by Dizman et al. also had comparingly high glass transition temperature of $169^{\circ} \mathrm{C}$ for the homopolymer [37]. Unfortunately only the quaternized polymers, that were water soluble and had no observable $T_{\mathrm{G}}$, exhibited good antimicrobial properties. 
(A)

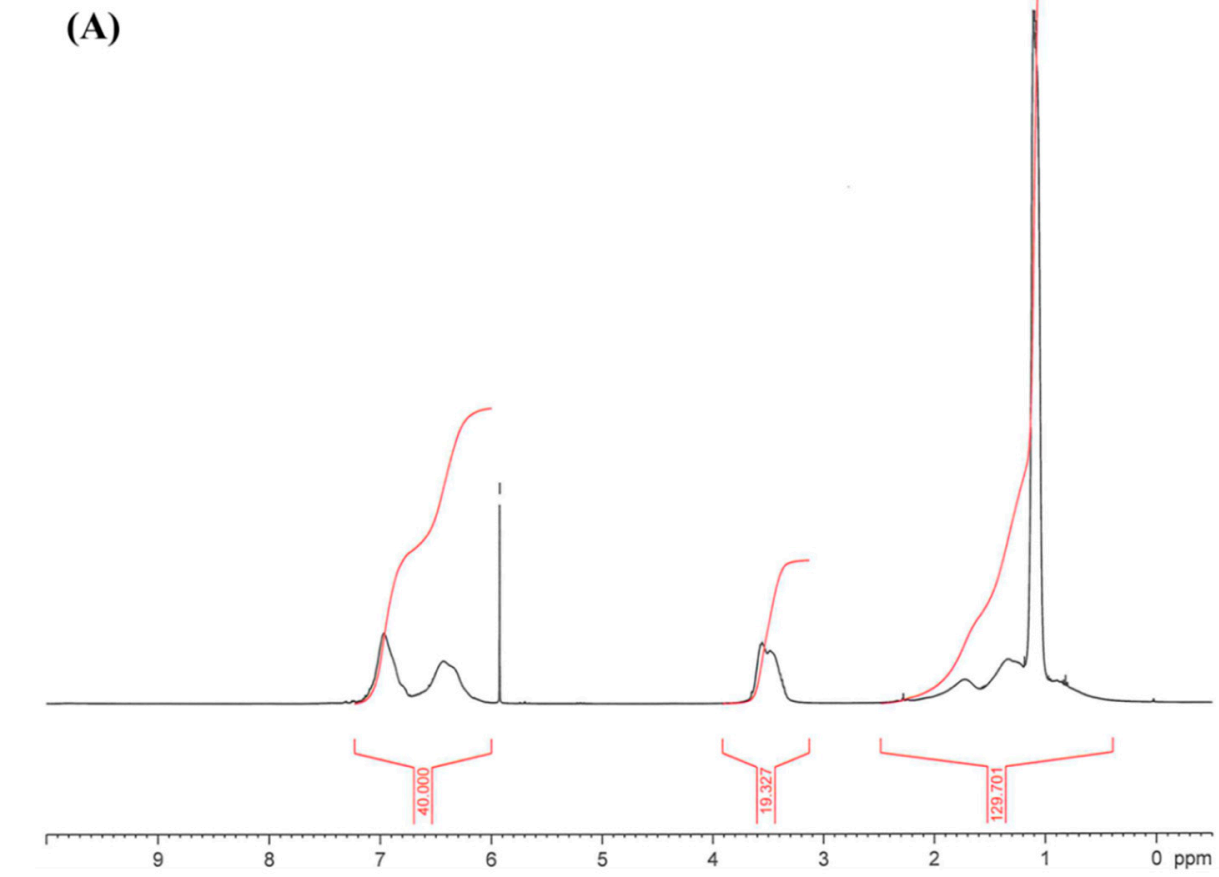

(B)

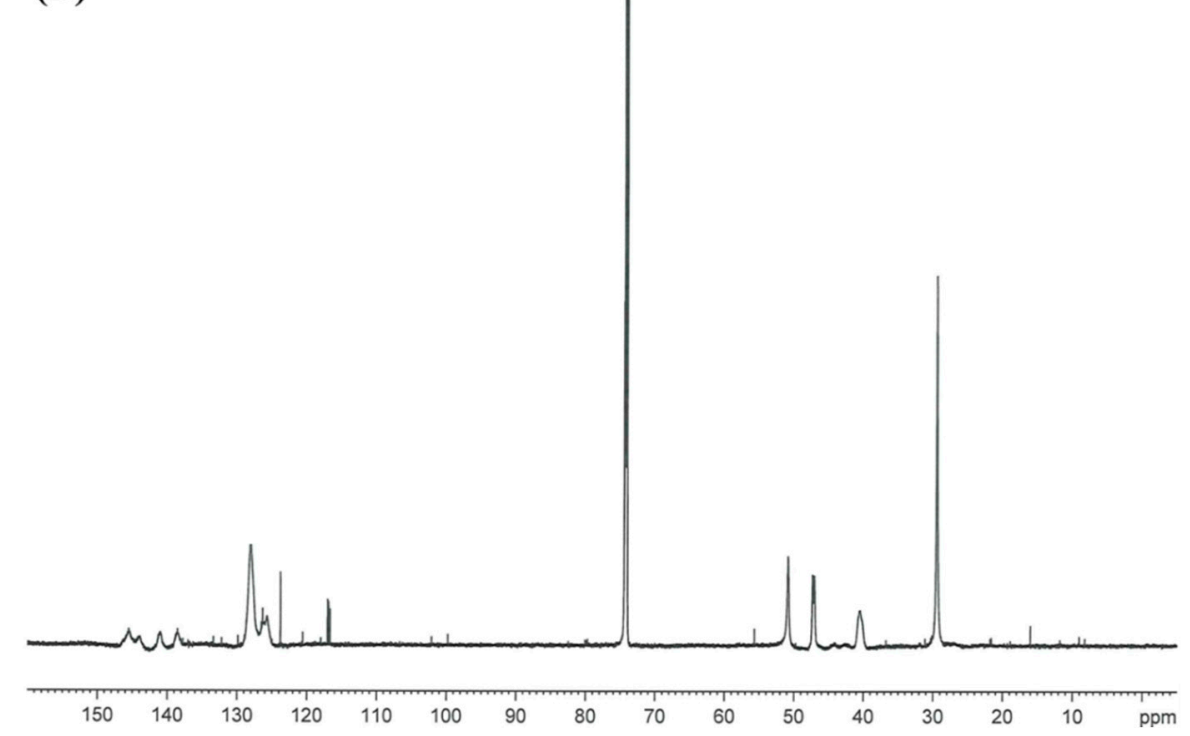

Figure 7. (A) ${ }^{1} \mathrm{H}$ NMR of poly(TBAMS); (B) ${ }^{13} \mathrm{C}$ NMR of poly(TBAMS).

\subsection{Laboratory Tests According to JIS Z 2801}

The antimicrobial activity of the polymer was analyzed by the test method JIS Z 2801:2000. The test method is based on the comparison of bacterial counts on reference and sample material after inoculation and incubation. The initial bacterial concentration of $S$. aureus determined on references samples without incubation was $1.6 \times 10^{5} \mathrm{CFU} / \mathrm{mL}$ and for $E$. coli was $4 \times 10^{5} \mathrm{CFU} / \mathrm{mL}$.

The arithmetic mean of bacterial counts of $S$. aureus on reference dishes is $5.5 \log _{10} \mathrm{CFU} / \mathrm{mL}$ and for E. coli is $6.5 \log _{10} \mathrm{CFU} / \mathrm{mL}$ after $2 \mathrm{~h}$ incubation time at $35{ }^{\circ} \mathrm{C}$. Bacterial counts of $S$. aureus and E. coli on poly(TBAMS) were below the detection limit of $1 \log _{10} \mathrm{CFU} / \mathrm{mL}$ and show a reduction level of $4.5 \log _{10}$ units for $S$. aureus and $5.5 \log _{10}$ units for $E$. coli respectively. Thus, the antimicrobial activity 
is proven against $S$. aureus and E. coli on poly(TBAMS). Poly(TBAMS) shows excellent antimicrobial properties towards the investigated microorganisms.

\subsection{Incubation of Poly(TBAMS) in E. coli Culture for Scanning Electron Microscopy (SEM) Imaging}

SEM was applied in order to investigate morphological changes of bacteria in contact with poly(TBAMS). In the case of the gram negative E. coli after $12 \mathrm{~h}$ of contact pronounced morphological changes were observed, whereas cells in the blank petri dishes remained unchanged. Furthermore the cells show a exudate of fibrous and granular material after incubation with poly(TBAMS). The surface of treated bacteria appears to be wrinkled and rough if compared with untreated cells (Figure 8). Additionally, a lot of debris was observed in the treated E. coli. Most likely the debris was formed due the lysis of cells.
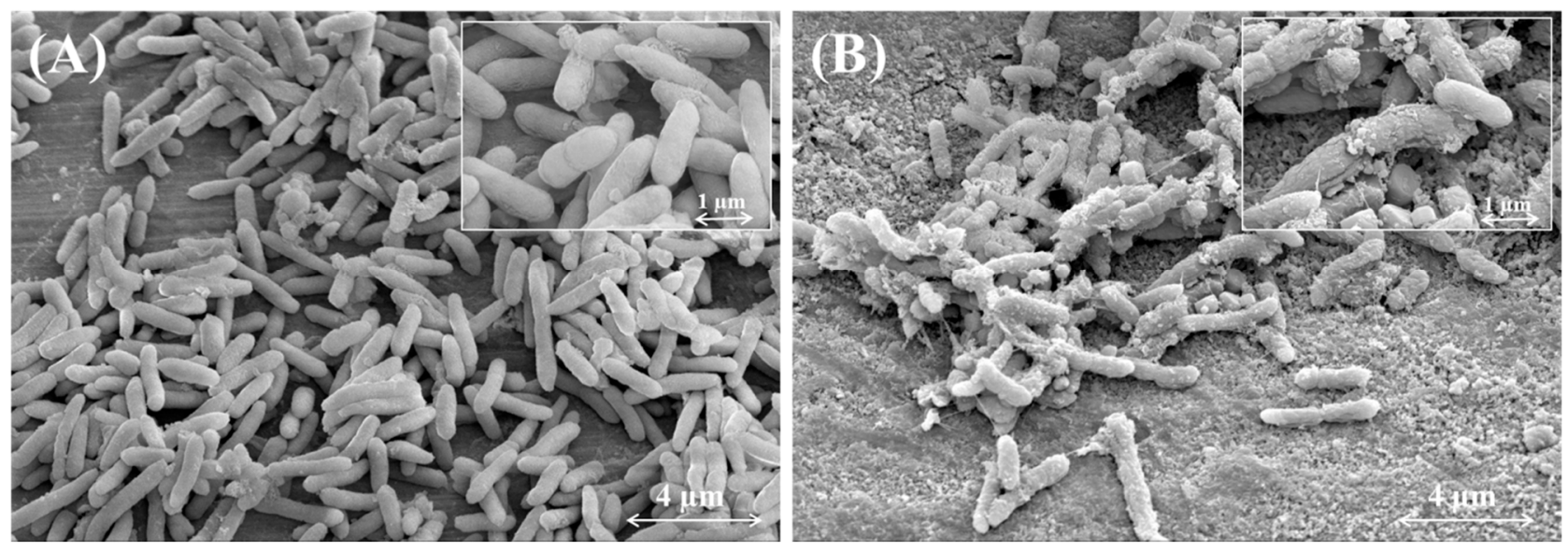

Figure 8. (A) E. coli after $12 \mathrm{~h}$ incubation in blank petri dish; (B) E. coli after $12 \mathrm{~h}$ incubation in petri dish coated with poly(TBAMS).

The SEM images gave striking evidence that the mode of action of poly(TBAMS) is most likely due to disruption of cell walls. Very similar observations, concerning the morphological changes, have been made in the case of poly(TBAEMA) grafted on polyethylene [38], a poly ( $N, N$-dimethyl-aminomethylstyrene) bonded to a polystyrene fiber [39] and water soluble $\beta$-Chitosan [40].

\section{Experimental Section}

All chemicals were of analytical grade or higher. Vinylbenzyl chloride was purchased from Dow chemicals as a mixture of isomers ( $57 \%$ meta, $43 \%$ para).

Melting points were recorded on a SPM30 (Stuart, Staffordshire, UK) using open capillaries. GC-MS were recorded on a QP2010plus (Shimadzu, Duisburg, Germany) using a FS supreme 5 column (30 m, ID $0.25 \mathrm{~mm}$, film $0.25 \mathrm{~mm}$ ). Detection was performed at $70 \mathrm{eV}$ in EI mode. $\mathrm{APCI}^{+}$-MS were recorded on a Bruker esquire 4000 (Bruker Daltonics, Bremen, Germany) instrument. Infrared spectra were recorded on a spectrum one (Perkin Elmer, Waltham, MA, USA) spectrometer using ATR technique. Differential scanning calorimetry analysis was carried out on a DSC821 (Mettler Toledo, Greifensee, Swiss) system. ${ }^{1} \mathrm{H}$ NMR and ${ }^{13} \mathrm{C}$ NMR spectra were acquired on a Bruker Avance III NMR (Bruker BipSpin, Rheinstetten, Germany) spectrometer with an observing frequency of 
$400 \mathrm{MHz}$ for ${ }^{1} \mathrm{H}$ and $100 \mathrm{MHz}$ for ${ }^{13} \mathrm{C}$, respectively. The samples were measured on the delta $(\delta)$ scale and are referenced to Trichloroethane $\left({ }^{1} \mathrm{H} \delta 5.93 \mathrm{ppm},{ }^{13} \mathrm{C} \delta 74.2 \mathrm{ppm}\right)$ that was used as solvent.

\subsection{Synthesis of Aklylaminomethylstyrenes}

In a $1 \mathrm{~L}$ round bottom flask equipped with a reflux condenser and dripping funnel there were placed $\mathrm{H}_{2} \mathrm{O}(200 \mathrm{~mL})$ and $\mathrm{NaOH}(42 \mathrm{~g}, 1.05 \mathrm{~mol})$. After complete dissolution of the $\mathrm{NaOH}$ the solution was cooled to $25{ }^{\circ} \mathrm{C}$ the amine $(1.05 \mathrm{~mol})$ was added. The apparatus was put under inert atmosphere by purging with nitrogen. The heterogeneous mixture was heated to $70{ }^{\circ} \mathrm{C}$ using an external oil bath and chloromethylstyrene $(53.42 \mathrm{~g}, 0.35 \mathrm{~mol})$ in THF $(150 \mathrm{~mL})$ was added drop wise over the course of $75 \mathrm{~min}$. After the addition was complete the reaction was stirred au $70{ }^{\circ} \mathrm{C}$ for additional $24 \mathrm{~h}$. The organic phase was separated, dried over $\mathrm{NaSO}_{4}$ and filtered through a sintered glass funnel. Volatiles were removed under reduced pressure $\left(30 \mathrm{mbar}, 40{ }^{\circ} \mathrm{C}\right)$. Further workup was performed using fractional distillation.

Propyaminomethylstyrene (1): Distillation: $135-140{ }^{\circ} \mathrm{C}, 20$ mbar. Yield: $41.57 \mathrm{~g}$ (65\%). Purity: 96\% (GC/MS, total area). Analysis: EI-MS: $\mathrm{M}^{+} 175 \mathrm{~m} / \mathrm{z}, 146 \mathrm{~m} / \mathrm{z}, 117 \mathrm{~m} / \mathrm{z}, 91 \mathrm{~m} / \mathrm{z}, 58 \mathrm{~m} / \mathrm{z}, 39 \mathrm{~m} / \mathrm{z}$. IR: $3309 \mathrm{~cm}^{-1} \cdot \mathrm{w}, 3087 \mathrm{~cm}^{-1} \cdot \mathrm{w}, 3006 \mathrm{~cm}^{-1} \cdot \mathrm{w}, 2953 \mathrm{~cm}^{-1} \cdot \mathrm{m}, 2930 \mathrm{~cm}^{-1} \cdot \mathrm{m}, 2873 \mathrm{~cm}^{-1} \cdot \mathrm{m}$, $1629 \mathrm{~cm}^{-1} \cdot \mathrm{m}, 1602 \mathrm{~cm}^{-1} \cdot \mathrm{w}, 1582 \mathrm{~cm}^{-1} \cdot \mathrm{w}, 1510 \mathrm{~cm}^{-1} \cdot \mathrm{m}, 1456 \mathrm{~cm}^{-1} \cdot \mathrm{s}, 1120 \mathrm{~cm}^{-1} \cdot \mathrm{s}, 989 \mathrm{~cm}^{-1} \cdot \mathrm{s}$, $903 \mathrm{~cm}^{-1} \cdot \mathrm{s}, 796 \mathrm{~cm}^{-1} \cdot \mathrm{s}, 712 \mathrm{~cm}^{-1} \cdot \mathrm{s}$.

Isopropylaminomethylstyrene (2): Distillation: $85-93{ }^{\circ} \mathrm{C}, 1$ mbar. Yield: $52.82 \mathrm{~g}$ (81\%). Purity: 95\% (GC/MS, total area). Analysis: EI-MS: $\mathrm{M}^{+} 175 \mathrm{~m} / \mathrm{z}, 160 \mathrm{~m} / \mathrm{z}, 117 \mathrm{~m} / \mathrm{z}, 91 \mathrm{~m} / \mathrm{z}, 65 \mathrm{~m} / \mathrm{z}, 30 \mathrm{~m} / \mathrm{z}$.

IR: $3310 \mathrm{~cm}^{-1} \cdot \mathrm{w}, 3087 \mathrm{~cm}^{-1} \cdot \mathrm{w}, 2963 \mathrm{~cm}^{-1} \cdot \mathrm{s}, 2869 \mathrm{~cm}^{-1} \cdot \mathrm{m}, 2825 \mathrm{~cm}^{-1} \cdot \mathrm{w}, 1630 \mathrm{~cm}^{-1} \cdot \mathrm{m}$, $1602 \mathrm{~cm}^{-1} \cdot \mathrm{w}, 1582 \mathrm{~cm}^{-1} \cdot \mathrm{w}, 1510 \mathrm{~cm}^{-1} \cdot \mathrm{m}, 1468 \mathrm{~cm}^{-1} \cdot \mathrm{m}, 1439 \mathrm{~cm}^{-1} \cdot \mathrm{m}, 1405 \mathrm{~cm}^{-1} \cdot \mathrm{m}, 1379 \mathrm{~cm}^{-1} \cdot \mathrm{s}$, $1337 \mathrm{~cm}^{-1} \cdot \mathrm{m}, 1171 \mathrm{~cm}^{-1} \cdot \mathrm{s}, 1124 \mathrm{~cm}^{-1} \cdot \mathrm{m}, 1071 \mathrm{~cm}^{-1} \cdot \mathrm{m}, 989 \mathrm{~cm}^{-1} \cdot \mathrm{s}, 903 \mathrm{~cm}^{-1} \cdot \mathrm{s}, 828 \mathrm{~cm}^{-1} \cdot \mathrm{s}$, $796 \mathrm{~cm}^{-1} \cdot \mathrm{s}, 712 \mathrm{~cm}^{-1} \cdot \mathrm{s}$.

Butylaminomethylstyrene (3): Distillation $150-153{ }^{\circ} \mathrm{C}, 20$ mbar. Yield: $46.06 \mathrm{~g}$ (66\%). Purity: 96\% (GC/MS, total area). Analysis: EI-MS: $\mathrm{M}^{+} 189 \mathrm{~m} / \mathrm{z}, 146 \mathrm{~m} / \mathrm{z}, 117 \mathrm{~m} / \mathrm{z}, 91 \mathrm{~m} / \mathrm{z}, 65 \mathrm{~m} / \mathrm{z}, 29 \mathrm{~m} / \mathrm{z}$.

IR: $3309 \mathrm{~cm}^{-1} \cdot \mathrm{w}, 3807 \mathrm{~cm}^{-1} \cdot \mathrm{w}, 3007 \mathrm{~cm}^{-1} \cdot \mathrm{w}, 2955 \mathrm{~cm}^{-1} \cdot \mathrm{m}, 2927 \mathrm{~cm}^{-1} \cdot \mathrm{w}, 1582 \mathrm{~cm}^{-1} \cdot \mathrm{w}$, $510 \mathrm{~cm}^{-1} \cdot \mathrm{m}, 1456 \mathrm{~cm}^{-1} \cdot \mathrm{s}, 1405 \mathrm{~cm}^{-1} \cdot \mathrm{m}, 1377 \mathrm{~cm}^{-1} \cdot \mathrm{w}, 1120 \mathrm{~cm}^{-1} \cdot \mathrm{s}, 988 \mathrm{~cm}^{-1} \cdot \mathrm{s}, 903 \mathrm{~cm}^{-1} \cdot \mathrm{s}$, $825 \mathrm{~cm}^{-1} \cdot \mathrm{s}, 796 \mathrm{~cm}^{-1} \cdot \mathrm{s}, 712 \mathrm{~cm}^{-1} \cdot \mathrm{s}$.

Sec-Butylaminomethylstyrole (4): Distillation: $140-145^{\circ} \mathrm{C}, 21$ mbar. Yield: $53.21 \mathrm{~g}(76 \%)$. Purity: 95\% (GC/MS, total area). Analysis: EI-MS: $\mathrm{M}^{+} 189 \mathrm{~m} / \mathrm{z}, 174 \mathrm{~m} / \mathrm{z}, 160 \mathrm{~m} / \mathrm{z}, 117 \mathrm{~m} / \mathrm{z}, 91 \mathrm{~m} / \mathrm{z}, 65 \mathrm{~m} / \mathrm{z}, 39 \mathrm{~m} / \mathrm{z}$.

IR: $3320 \mathrm{~cm}^{-1} \cdot \mathrm{w}, 3087 \mathrm{~cm}^{-1} \cdot \mathrm{w}, 2961 \mathrm{~cm}^{-1} \cdot \mathrm{s}, 2928 \mathrm{~cm}^{-1} \cdot \mathrm{m}, 2875 \mathrm{~cm}^{-1} \cdot \mathrm{m}, 1630 \mathrm{~cm}^{-1} \cdot \mathrm{m}$, $1602 \mathrm{~cm}^{-1} \cdot \mathrm{w}, 1528 \mathrm{~cm}^{-1} \cdot \mathrm{w}, 1510 \mathrm{~cm}^{-1} \cdot \mathrm{m}, 1462 \mathrm{~cm}^{-1} \cdot \mathrm{s}, 1405 \mathrm{~cm}^{-1} \cdot \mathrm{m}, 1373 \mathrm{~cm}^{-1} \cdot \mathrm{m}, 1347 \mathrm{~cm}^{-1} \cdot \mathrm{m}$, $1166 \mathrm{~cm}^{-1} \cdot \mathrm{m}, 1079 \mathrm{~cm}^{-1} \cdot \mathrm{m}, 988 \mathrm{~cm}^{-1} \cdot \mathrm{s}, 903 \mathrm{~cm}^{-1} \cdot \mathrm{s}, 828 \mathrm{~cm}^{-1} \cdot \mathrm{s}, 796 \mathrm{~cm}^{-1} \cdot \mathrm{s}, 712 \mathrm{~cm}^{-1} \cdot \mathrm{s}$.

Isobutylaminomethylstyrole (5): Distillation: $145-14{ }^{\circ} \mathrm{C}, 24$ mbar. Yield: $51.55 \mathrm{~g}$ (74\%). Purity: 95\% (GC/MS, total area). Analysis: EI-MS: $\mathrm{M}^{+} 189 \mathrm{~m} / \mathrm{z}, 146 \mathrm{~m} / \mathrm{z}, 117 \mathrm{~m} / \mathrm{z}, 91 \mathrm{~m} / \mathrm{z}, 65 \mathrm{~m} / \mathrm{z}, 41 \mathrm{~m} / \mathrm{z}$.

IR: $3221 \mathrm{~cm}^{-1} \cdot \mathrm{w}, 3087 \mathrm{~cm}^{-1} \cdot \mathrm{w}, 3007 \mathrm{~cm}^{-1} \cdot \mathrm{w}, 2953 \mathrm{~cm}^{-1} \cdot \mathrm{s}, 2870 \mathrm{~cm}^{-1} \cdot \mathrm{m}, 2809 \mathrm{~cm}^{-1} \cdot \mathrm{m}$, $1630 \mathrm{~cm}^{-1} \cdot \mathrm{m}, 1602 \mathrm{~cm}^{-1} \cdot \mathrm{w}, 1582 \mathrm{~cm}^{-1} \cdot \mathrm{w}, 1510 \mathrm{~cm}^{-1} \cdot \mathrm{m}, 1462 \mathrm{~cm}^{-1} \cdot \mathrm{s}, 1444 \mathrm{~cm}^{-1} \cdot \mathrm{s}, 1405 \mathrm{~cm}^{-1} \cdot \mathrm{m}$, $1385 \mathrm{~cm}^{-1} \cdot \mathrm{m}, 1365 \mathrm{~cm}^{-1} \cdot \mathrm{m}, 1109 \mathrm{~cm}^{-1} \cdot \mathrm{s}, 988 \mathrm{~cm}^{-1} \cdot \mathrm{s}, 903 \mathrm{~cm}^{-1} \cdot \mathrm{s}, 796 \mathrm{~cm}^{-1} \cdot \mathrm{s}, 722 \mathrm{~cm}^{-1} \cdot \mathrm{s}$.

Pentylaminomethylstyrole (6): Distillation: $117-123{ }^{\circ} \mathrm{C}, 1$ mbar. Yield: $56.42 \mathrm{~g}$ (76\%). Purity: 96\% (GC/MS, total area). Analysis: EI-MS: $\mathrm{M}^{+} 203 \mathrm{~m} / \mathrm{z}, 146 \mathrm{~m} / \mathrm{z}, 117 \mathrm{~m} / \mathrm{z}, 43 \mathrm{~m} / \mathrm{z}, 29 \mathrm{~m} / \mathrm{z}$. 
IR: $3309 \mathrm{~cm}^{-1} \cdot \mathrm{w}, 3087 \mathrm{~cm}^{-1} \cdot \mathrm{w}, 3007 \mathrm{~cm}^{-1} \cdot \mathrm{w}, 2954 \mathrm{~cm}^{-1} \cdot \mathrm{s}, 2915 \mathrm{~cm}^{-1} \cdot \mathrm{s}, 2868 \mathrm{~cm}^{-1} \cdot \mathrm{m}$, $2816 \mathrm{~cm}^{-1} \cdot \mathrm{m}, 1630 \mathrm{~cm}^{-1} \cdot \mathrm{m}, 1602 \mathrm{~cm}^{-1} \cdot \mathrm{w}, 1582 \mathrm{~cm}^{-1} \cdot \mathrm{w}, 1510 \mathrm{~cm}^{-1} \cdot \mathrm{m}, 1463 \mathrm{~cm}^{-1} \cdot \mathrm{s}, 1405 \mathrm{~cm}^{-1} \cdot \mathrm{m}$, $1383 \mathrm{~cm}^{-1} \cdot \mathrm{m}, 1366 \mathrm{~cm}^{-1} \cdot \mathrm{m}, 1110 \mathrm{~cm}^{-1} \cdot \mathrm{s}, 988 \mathrm{~cm}^{-1} \cdot \mathrm{s}, 903 \mathrm{~cm}^{-1} \cdot \mathrm{s}, 795 \mathrm{~cm}^{-1} \cdot \mathrm{s}, 712 \mathrm{~cm}^{-1} \cdot \mathrm{s}$.

3-Pentylaminomethylstyrole (7): Distillation: $120-123{ }^{\circ} \mathrm{C}, 1$ mbar. Yield: $59.65 \mathrm{~g}$ (76\%). Purity: 95\% (GC/MS, total area). Analysis: EI-MS: $\mathrm{M}^{+} 203 \mathrm{~m} / \mathrm{z}, 146 \mathrm{~m} / \mathrm{z}, 117 \mathrm{~m} / \mathrm{z}, 91 \mathrm{~m} / \mathrm{z}, 41 \mathrm{~m} / \mathrm{z}$.

IR: $3336 \mathrm{~cm}^{-1} \cdot \mathrm{w}, 3088 \mathrm{~cm}^{-1} \cdot \mathrm{w}, 2960 \mathrm{~cm}^{-1} \cdot \mathrm{m}, 2929 \mathrm{~cm}^{-1} \cdot \mathrm{m}, 2873 \mathrm{~cm}^{-1} \cdot \mathrm{m}, 1630 \mathrm{~cm}^{-1} \cdot \mathrm{m}$, $1602 \mathrm{~cm}^{-1} \cdot \mathrm{w}, 1582 \mathrm{~cm}^{-1} \cdot \mathrm{w}, 1510 \mathrm{~cm}^{-1} \cdot \mathrm{m}, 1549 \mathrm{~cm}^{-1} \cdot \mathrm{s}, 1405 \mathrm{~cm}^{-1} \cdot \mathrm{m}, 1379 \mathrm{~cm}^{-1} \cdot \mathrm{m}, 1159 \mathrm{~cm}^{-1} \cdot \mathrm{m}$, $1085 \mathrm{~cm}^{-1} \cdot \mathrm{m}, 989 \mathrm{~cm}^{-1} \cdot \mathrm{s}, 903 \mathrm{~cm}^{-1} \cdot \mathrm{s}, 796 \mathrm{~cm}^{-1} \cdot \mathrm{s}, 713 \mathrm{~cm}^{-1} \cdot \mathrm{s}$.

Isopentylaminomethylstyrole (8): Distillation: $140-145{ }^{\circ} \mathrm{C}, 9$ mbar. Yield: $50.93 \mathrm{~g}$ (76\%). Purity: 96\% (GC/MS, total area). Analysis: EI-MS: $\mathrm{M}^{+} 203 \mathrm{~m} / \mathrm{z}, 146 \mathrm{~m} / \mathrm{z}, 117 \mathrm{~m} / \mathrm{z}, 41 \mathrm{~m} / \mathrm{z}$.

IR: $3309 \mathrm{~cm}^{-1} \cdot \mathrm{w}, 3087 \mathrm{~cm}^{-1} \cdot \mathrm{w}, 3007 \mathrm{~cm}^{-1} \cdot \mathrm{w}, 2954 \mathrm{~cm}^{-1} \cdot \mathrm{s}, 2915 \mathrm{~cm}^{-1} \cdot \mathrm{s}, 2868 \mathrm{~cm}^{-1} \cdot \mathrm{m}$, $2816 \mathrm{~cm}^{-1} \cdot \mathrm{m}, 1630 \mathrm{~cm}^{-1} \cdot \mathrm{m}, 1602 \mathrm{~cm}^{-1} \cdot \mathrm{w}, 1582 \mathrm{~cm}^{-1} \cdot \mathrm{w}, 1510 \mathrm{~cm}^{-1} \cdot \mathrm{m}, 1463 \mathrm{~cm}^{-1} \cdot \mathrm{s}, 1405 \mathrm{~cm}^{-1} \cdot \mathrm{m}$, $1387 \mathrm{~cm}^{-1} \cdot \mathrm{m}, 1366 \mathrm{~cm}^{-1} \cdot \mathrm{m}, 1110 \mathrm{~cm}^{-1} \cdot \mathrm{s}, 988 \mathrm{~cm}^{-1} \cdot \mathrm{s}, 903 \mathrm{~cm}^{-1} \cdot \mathrm{s}, 796 \mathrm{~cm}^{-1} \cdot \mathrm{s}, 712 \mathrm{~cm}^{-1} \cdot \mathrm{s}$.

Tert-pentylaminomethylstyrole (9): Distillation: $105-110{ }^{\circ} \mathrm{C}, 1$ mbar. Yield: $55.12 \mathrm{~g}$ (74\%). Purity: 96\% (GC/MS, total area). Analysis: EI-MS: $\mathrm{M}^{+} 203 \mathrm{~m} / \mathrm{z}, 188 \mathrm{~m} / \mathrm{z}, 174 \mathrm{~m} / \mathrm{z}, 117 \mathrm{~m} / \mathrm{z}, 91 \mathrm{~m} / \mathrm{z}, 42 \mathrm{~m} / \mathrm{z}$.

IR: $3320 \mathrm{~cm}^{-1} \cdot \mathrm{w}, 3087 \mathrm{~cm}^{-1} \cdot \mathrm{w}, 2963 \mathrm{~cm}^{-1} \cdot \mathrm{s}, 2887 \mathrm{~cm}^{-1} \cdot \mathrm{s}, 1630 \mathrm{~cm}^{-1} \cdot \mathrm{m}, 1602 \mathrm{~cm}^{-1} \cdot \mathrm{w}$, $1582 \mathrm{~cm}^{-1} \cdot \mathrm{w}, 1510 \mathrm{~cm}^{-1} \cdot \mathrm{m}, 1460 \mathrm{~cm}^{-1} \cdot \mathrm{s}, 1405 \mathrm{~cm}^{-1} \cdot \mathrm{m}, 1380 \mathrm{~cm}^{-1} \cdot \mathrm{m}, 1363 \mathrm{~cm}^{-1} \cdot \mathrm{m}, 1289 \mathrm{~cm}^{-1} \cdot \mathrm{s}$, $1201 \mathrm{~cm}^{-1} \cdot \mathrm{s}, 1093 \mathrm{~cm}^{-1} \cdot \mathrm{s}, 988 \mathrm{~cm}^{-1} \cdot \mathrm{s}, 903 \mathrm{~cm}^{-1} \cdot \mathrm{s}, 827 \mathrm{~cm}^{-1} \cdot \mathrm{s}, 796 \mathrm{~cm}^{-1} \cdot \mathrm{s}, 712 \mathrm{~cm}^{-1} \cdot \mathrm{s}$.

Cyclopentylaminomethylstyrole (10): Distillation: $132-140{ }^{\circ} \mathrm{C}, 1$ mbar. Yield: $51.2 \mathrm{~g}$ (70\%). Purity: 96\% (GC/MS, total area). Analysis: EI-MS: $\mathrm{M}^{+} 201 \mathrm{~m} / \mathrm{z}, 172 \mathrm{~m} / \mathrm{z}, 158 \mathrm{~m} / \mathrm{z}, 117 \mathrm{~m} / \mathrm{z}, 91 \mathrm{~m} / \mathrm{z}$, $84 \mathrm{~m} / \mathrm{z}, 65 \mathrm{~m} / \mathrm{z}, 41 \mathrm{~m} / \mathrm{z}$.

IR: $3315 \mathrm{~cm}^{-1} \cdot \mathrm{w}, 3086 \mathrm{~cm}^{-1} \cdot \mathrm{w}, 3006 \mathrm{~cm}^{-1} \cdot \mathrm{w}, 2924 \mathrm{~cm}^{-1} \cdot \mathrm{s}, 2851 \mathrm{~cm}^{-1} \cdot \mathrm{s}, 1630 \mathrm{~cm}^{-1} \cdot \mathrm{m}$, $1602 \mathrm{~cm}^{-1} \cdot \mathrm{w}, 1582 \mathrm{~cm}^{-1} \cdot \mathrm{w}, 1510 \mathrm{~cm}^{-1} \cdot \mathrm{m}, 1448 \mathrm{~cm}^{-1} \cdot \mathrm{s}, 1405 \mathrm{~cm}^{-1} \cdot \mathrm{m}, 1360 \mathrm{~cm}^{-1} \cdot \mathrm{m}, 1346 \mathrm{~cm}^{-1} \cdot \mathrm{m}$, $1121 \mathrm{~cm}^{-1} \cdot \mathrm{s}, 988 \mathrm{~cm}^{-1} \cdot \mathrm{s}, 903 \mathrm{~cm}^{-1} \cdot \mathrm{s}, 826 \mathrm{~cm}^{-1} \cdot \mathrm{s}, 796 \mathrm{~cm}^{-1} \cdot \mathrm{s}, 712 \mathrm{~cm}^{-1} \cdot \mathrm{s}$.

Cyclohexylaminomethylstyrole (11): Distillation: $135-137^{\circ} \mathrm{C}, 1$ mbar. Yield: $62.75 \mathrm{~g}$ (80\%). Purity: 95\% (GC/MS, total area). Analysis: EI-MS: $\mathrm{M}^{+} 215 \mathrm{~m} / \mathrm{z}, 172 \mathrm{~m} / \mathrm{z}, 158 \mathrm{~m} / \mathrm{z}, 117 \mathrm{~m} / \mathrm{z}, 98 \mathrm{~m} / \mathrm{z}$, $91 \mathrm{~m} / \mathrm{z}, 55 \mathrm{~m} / \mathrm{z}, 41 \mathrm{~m} / \mathrm{z}$.

IR: $3310 \mathrm{~cm}^{-1} \cdot \mathrm{w}, 3086 \mathrm{~cm}^{-1} \cdot \mathrm{w}, 3006 \mathrm{~cm}^{-1} \cdot \mathrm{w}, 2924 \mathrm{~cm}^{-1} \cdot \mathrm{s}, 2851 \mathrm{~cm}^{-1} \cdot \mathrm{s}, 1630 \mathrm{~cm}^{-1} \cdot \mathrm{m}$, $1602 \mathrm{~cm}^{-1} \cdot \mathrm{w}, 1581 \mathrm{~cm}^{-1} \cdot \mathrm{w}, 1510 \mathrm{~cm}^{-1} \cdot \mathrm{m}, 1448 \mathrm{~cm}^{-1} \cdot \mathrm{s}, 1405 \mathrm{~cm}^{-1} \cdot \mathrm{s}, 1360 \mathrm{~cm}^{-1} \cdot \mathrm{m}, 1346 \mathrm{~cm}^{-1} \cdot \mathrm{m}$, $1124 \mathrm{~cm}^{-1} \cdot \mathrm{s}, 988 \mathrm{~cm}^{-1} \cdot \mathrm{s}, 903 \mathrm{~cm}^{-1} \cdot \mathrm{s}, 826 \mathrm{~cm}^{-1} \cdot \mathrm{s}, 795 \mathrm{~cm}^{-1} \cdot \mathrm{s}, 712 \mathrm{~cm}^{-1} \cdot \mathrm{s}$.

Tetramethylpiperidinaminomethylstyrole (12): Distillation: $165-170{ }^{\circ} \mathrm{C}, 2$ mbar. Yield: $60.60 \mathrm{~g}$ (60\%). Purity: 95\% (GC/MS, total area). Analysis: EI-MS: $\mathrm{M}^{+} 272 \mathrm{~m} / \mathrm{z}, 257 \mathrm{~m} / \mathrm{z}, 215 \mathrm{~m} / \mathrm{z}, 173 \mathrm{~m} / \mathrm{z}$, $155 \mathrm{~m} / \mathrm{z}, 117 \mathrm{~m} / \mathrm{z}, 98 \mathrm{~m} / \mathrm{z}, 58 \mathrm{~m} / \mathrm{z}, 41 \mathrm{~m} / \mathrm{z}$.

IR: $3305 \mathrm{~cm}^{-1} \cdot \mathrm{w}, 3085 \mathrm{~cm}^{-1} \cdot \mathrm{w}, 2954 \mathrm{~cm}^{-1} \cdot \mathrm{s}, 2917 \mathrm{~cm}^{-1} \cdot \mathrm{s}, 1630 \mathrm{~cm}^{-1} \cdot \mathrm{m}, 1601 \mathrm{~cm}^{-1} \cdot \mathrm{w}$, $1582 \mathrm{~cm}^{-1} \cdot \mathrm{w}, 1510 \mathrm{~cm}^{-1} \cdot \mathrm{m}, 1454 \mathrm{~cm}^{-1} \cdot \mathrm{m}, 1374 \mathrm{~cm}^{-1} \cdot \mathrm{s}, 1363 \mathrm{~cm}^{-1} \cdot \mathrm{s}, 1237 \mathrm{~cm}^{-1} \cdot \mathrm{s}, 1197 \mathrm{~cm}^{-1} \cdot \mathrm{s}$, $1107 \mathrm{~cm}^{-1} \cdot \mathrm{s}, 989 \mathrm{~cm}^{-1} \cdot \mathrm{s}, 904 \mathrm{~cm}^{-1} \cdot \mathrm{s}, 826 \mathrm{~cm}^{-1} \cdot \mathrm{m}, 795 \mathrm{~cm}^{-1} \cdot \mathrm{s}, 712 \mathrm{~cm}^{-1} \cdot \mathrm{s}$.

\subsection{Polymerization of the Aklylaminomethylstyrenes}

In a $250 \mathrm{~mL}$ round bottom flask the corresponding monomer $(145.8 \mathrm{mmol})$ were dissolved in ethanol $(60 \mathrm{~mL})$, a solution of AIBN $(0.37 \mathrm{~g})$ in 2-butanone $(10 \mathrm{~mL})$ was added. The apparatus was put under inert atmosphere by purging with nitrogen. The contents of the flask were heated under constant stirring 
(300 rpm) to $60{ }^{\circ} \mathrm{C}$ with internal temperature control in a silicon oil bath for $48 \mathrm{~h}$. The polymer was precipitated by slowly dripping the contents of the flask in to a stirred bath of $5 \mathrm{~L} \mathrm{H}_{2} \mathrm{O}$. The solids were removed by filtration. Removal of residual monomers and oligomers was accomplished by boiling the polymer $(20 \mathrm{~g})$ in aqueous isoporopyl alcohol $(100 \mathrm{~mL}, 5 \%(v / v))$. Finally, the polymer was dried in a vacuum drying cabinet (Binder VD23, Tuttlingen, Germany) at $140{ }^{\circ} \mathrm{C}, 1 \mathrm{mbar}$ until no more change in polymer mass was observed. Water soluble polymers were directly dried in vacuo after removal of volatiles $\left(40^{\circ} \mathrm{C}, 1 \mathrm{mbar}\right)$ without prior precipitation in water.

\subsection{Synthesis of TBAMS}

In a $1 \mathrm{~L}$ three neck round bottom flask, equipped with reflux condenser, dripping funnel and magnetic stir bar $\mathrm{H}_{2} \mathrm{O}(300 \mathrm{~mL})$ and $\mathrm{NaOH}(36 \mathrm{~g}, 0.9 \mathrm{~mol})$ were placed. The solution was cooled down to room temperature and tert-butyl amine $(65.82 \mathrm{~g}, 0.9 \mathrm{~mol})$ was added. The apparatus was put under inert atmosphere by purging with nitrogen. The contents of the flask were heated to $60{ }^{\circ} \mathrm{C}$ with internal temperature control in a silicon oil bath. Vinylbenzyl chloride $(45.78 \mathrm{~g}, 0.3 \mathrm{~mol})$ in THF (200 mL) was added in a drop wise manner over the course of $4 \mathrm{~h}$. Stirring and heating was continued over $20 \mathrm{~h}$. After this the organic phase was separated using an extraction funnel. It was washed with $\mathrm{H}_{2} \mathrm{O}(2 \times 50 \mathrm{~mL})$ and dried over $\mathrm{MgSO}_{4}$. TBAMS was isolated by fractional distillation $\left(105-114{ }^{\circ} \mathrm{C}, 2 \mathrm{mbar}\right)$ as colorless oil with a high refractive index. The yield was $55.65 \mathrm{~g}$ (96\%). Purity was 97\% (GC-MS, total area).

Analysis: EI-MS: $\mathrm{M}^{+} 189 \mathrm{~m} / \mathrm{z}, 1.08 \% ; 174 \mathrm{~m} / \mathrm{z}, 26.23 \% ; 117 \mathrm{~m} / \mathrm{z} 100.00 \% ; 91 \mathrm{~m} / \mathrm{z}, 9.06 \% ; 57 \mathrm{~m} / \mathrm{z}$, $3.76 \%$; $41 \mathrm{~m} / z, 6.92 \%$. IR: $3306 \mathrm{~cm}^{-1} \cdot \mathrm{w}, 3088 \mathrm{~cm}^{-1} \cdot \mathrm{w}, 2963 \mathrm{~cm}^{-1} \cdot \mathrm{s}, 1630 \mathrm{~cm}^{-1} \cdot \mathrm{m}, 1510 \mathrm{~cm}^{-1} \cdot \mathrm{m}$, $1479 \mathrm{~cm}^{-1} \cdot \mathrm{s}, 1307 \mathrm{~cm}^{-1} \cdot \mathrm{m}, 1228 \mathrm{~cm}^{-1} \cdot \mathrm{s} ; 1211 \mathrm{~cm}^{-1} \cdot \mathrm{s}, 988 \mathrm{~cm}^{-1} \cdot \mathrm{s}, 903 \mathrm{~cm}^{-1} \cdot \mathrm{s}, 827 \mathrm{~cm}^{-1} \cdot \mathrm{s}$, $797 \mathrm{~cm}^{-1} \cdot \mathrm{s}, 712 \mathrm{~cm}^{-1} .{ }^{1} \mathrm{H}$ NMR: $\delta 0.99 \mathrm{ppm}(\mathrm{s}, 1 \mathrm{H}, \mathrm{NH}), \delta 1.30 \mathrm{ppm}\left(\mathrm{s}, 9 \mathrm{H},-\left(\mathrm{CH}_{3}\right)_{3}\right), \delta 3.80 \mathrm{ppm}$ $\left(\mathrm{s}, 2 \mathrm{H},-\mathrm{CH}_{2}-\mathrm{NH}\right), \delta 5.35 \mathrm{ppm}$ and $\delta 5.87 \mathrm{ppm}\left(\mathrm{t}, 2 \mathrm{H},-\mathrm{CH}=\mathrm{CH}_{2}\right), \delta 6.85 \mathrm{ppm}\left(\mathrm{m}, 1 \mathrm{H},-\mathrm{CH}=\mathrm{CH}_{2}\right)$, $\delta 7,42 \mathrm{ppm}\left(\mathrm{m}, 4 \mathrm{H}\right.$, aromatic); ${ }^{13} \mathrm{C} \mathrm{NMR}: \delta 29.5 \mathrm{ppm}\left(\mathrm{C}-\left(\mathrm{CH}_{3}\right)\right), \delta 47,47.2 \mathrm{ppm}\left(-\mathrm{CH}_{2}-\mathrm{NH}\right)$, $\delta 50.7 \mathrm{ppm}\left(\mathrm{NH}-\mathrm{C}\left(\mathrm{CH}_{3}\right)_{3}\right), \delta 114.1 .7,113 \mathrm{ppm}\left(-\mathrm{CH}=\mathrm{CH}_{2}\right), \delta 124.9,126.4128 .1,128.8 \mathrm{ppm}$ (aromatic $\mathrm{CH}$ ), $\delta 126.4128 .6 \mathrm{ppm}$ (aromatic $\mathrm{CH}$ of para isomer), $\delta 136.3,136.9137 .2$, $137.8 \mathrm{ppm}$ (Quart-aromatic C), $\delta 141.8142 .3 \mathrm{ppm}\left(-\mathrm{CH}=\mathrm{CH}_{2}\right)$.

\subsection{Preparation of TBAMS Hydrochloride}

In a $500 \mathrm{~mL}$ round bottom flask TBAMS $(70 \mathrm{~g}, 0.369 \mathrm{~mol})$ in Acetone $(150 \mathrm{~mL})$ was placed. The flask was nestled in an ice bath and stirred magnetically. A slow stream of dry $\mathrm{HCl}$ (from $\mathrm{NaCl}$ and conc. $\mathrm{H}_{2} \mathrm{SO}_{4}$ ) was introduced via a glass capillary in to the solution until the solution was slightly acidic. Stirring was continued for $2 \mathrm{~h}$. After precipitation of the hydrochloride at $-20{ }^{\circ} \mathrm{C}$, the crystals were removed by filtration, washed with ice cold acetone $(2 \times 10 \mathrm{~mL})$ and dried in vacuum. The crystals were recrystallized from isopropyl alcohol/toluene $(10 \mathrm{~mL} / 150 \mathrm{~mL})$. After cooling to $-20{ }^{\circ} \mathrm{C}$ and drying in vacuum TBAMS·HCl (66.7 g, 80\% yield) was obtained.

Analysis: melting point: $172-174{ }^{\circ} \mathrm{C}$ decomp; IR: $2947 \mathrm{~cm}^{-1} \cdot \mathrm{s} ; 2745 \mathrm{~cm}^{-1} \cdot \mathrm{s}, 2430 \mathrm{~cm}^{-1} \cdot \mathrm{m}$; $1629 \mathrm{~cm}^{-1} \cdot \mathrm{m} ; 1589 \mathrm{~cm}^{-1} \cdot \mathrm{s} ; 1479 \mathrm{~cm}^{-1} \cdot \mathrm{m} ; 1447 \mathrm{~cm}^{-1} \cdot \mathrm{s} ; 1402 \mathrm{~cm}^{-1} \cdot \mathrm{s} ; 1367 \mathrm{~cm}^{-1} \cdot \mathrm{s} ; 1241 \mathrm{~cm}^{-1} \cdot \mathrm{m}$; $1198 \mathrm{~cm}^{-1} \cdot \mathrm{s} ; 985 \mathrm{~cm}^{-1} \cdot \mathrm{s} ; 924 \mathrm{~cm}^{-1} \cdot \mathrm{s} ; 808 \mathrm{~cm}^{-1} \cdot \mathrm{s} ; 711 \mathrm{~cm}^{-1} \cdot \mathrm{s} ; 570 \mathrm{~cm}^{-1} \cdot \mathrm{s}$. Elemental analysis: found: C 69.1\%; H 8.99\%, N 6.2\%, calculated for $\mathrm{C}_{13} \mathrm{H}_{20} \mathrm{ClN}$ : C 69.1\%, H 8.9\%, N 6.2\%. 


\subsection{Preparation of Poly(TBAMS)}

TBAMS (27 g, $0.14 \mathrm{~mol})$ was placed in a $500 \mathrm{~mL}$ two-neck round-bottom flask equipped with a reflux condenser (Duran glass, Germany). In a $30 \mathrm{~mL}$ glass crimp-top vial 2,2'-Azobisisobutyronitrile (AIBN, $0.37 \mathrm{~g}$ ) was dissolved in 2-butanone $(10 \mathrm{~mL})$. This initiator solution was added to the monomer, followed by ethanol $(150 \mathrm{~mL})$. The flask was transferred into an oil bath at room temperature (HBR 4 digital, IKA, Staufen, Germany) which was also used to stir the mixture via a magnetic stirrer. The apparatus was flushed with nitrogen (purity 99.99999\%) for at least $30 \mathrm{~min}$ to ensure an oxygen free atmosphere. The reaction solution was continuously stirred (approx. $300 \mathrm{rpm}$ ) during this time. Polymerization was carried out for $5 \mathrm{~h}$ at $55^{\circ} \mathrm{C}$ and additionally for $22.5 \mathrm{~h}$ at $65^{\circ} \mathrm{C}$. After the solution was cooled to room temperature, $\mathrm{H}_{2} \mathrm{O}(100 \mathrm{~mL})$ was slowly added via a $250 \mathrm{~mL}$ dropping funnel under stirring. After all water was added the stirrer was turned off and the solution stored for $12 \mathrm{~h}$ to separate the polymer from the upper ethanol phase which was removed by decantation. The precipitated polymer was dissolved in ethanol $(135 \mathrm{~mL})$ and the aforementioned precipitation process was repeated. The liquid phase is then analyzed via TLC (Silicagel 60, MeOH, UV detection). The polymer remained at the start, whereas the Monomer had a retention factor value of approximately 0.5 . The purification process was repeated until no more TBAMS can be observed via TLC. In a typical experiment, eight purification steps were necessary. The purified poly(TBAMS) solution was dried in a vacuum drying cabinet (Binder VD23, Tuttlingen, Germany) at $70{ }^{\circ} \mathrm{C}, 1 \mathrm{mbar}$ for $16 \mathrm{~h}$ and milled down using an ultra-centrifugal mill (ZM 200, Retsch, Haan, Germany). A $200 \mu \mathrm{m}$ sieve was applied, the mill is continuously cooled using liquid nitrogen. Yield: $20 \mathrm{~g}, 74 \%$.

GPC measurements were performed on a Viscotec GPC max VE2001 (Malvern Instruments, Malvern, United Kingdom) machine equipped with two PSS SD LIN M $5 \mu \mathrm{m} 8 \times 300 \mathrm{~mm}$ columns (Polymer Standards Services, Mainz, Germany) coupled in series using THF with $0.1 \%$ triethylamine as eluent and RI detection. Calibration was done with a Polystyrene standard (474-2520000 Da) (Polymer Standards Services, Mainz, Germany).

Analysis: $T_{\mathrm{G}}$ : $68^{\circ} \mathrm{C}$;GPC: Mw: $185588 \mathrm{Da}, \mathrm{Mn}: 3562 \mathrm{Da}, M \mathrm{w} / \mathrm{Mn}: 5.2$; IR: $2960 \mathrm{~cm}^{-1} \cdot \mathrm{s}$, $2921 \mathrm{~cm}^{-1} \cdot \mathrm{s}, 1605 \mathrm{~cm}^{-1} \cdot \mathrm{m}, 1443 \mathrm{~cm}^{-1} \cdot \mathrm{s}, 1360 \mathrm{~cm}^{-1} \cdot \mathrm{s}, 1228 \mathrm{~cm}^{-1} \cdot \mathrm{s}, 1088 \mathrm{~cm}^{-1} \cdot \mathrm{m}, 1019 \mathrm{~cm}^{-1} \cdot \mathrm{m}$, $810 \mathrm{~cm}^{-1} \cdot \mathrm{m}, 792 \mathrm{~cm}^{-1} \mathrm{~cm}, 704 \mathrm{~cm}^{-1} ;{ }^{1} \mathrm{HNMR}: \delta 0.4-2.5 \mathrm{ppm}\left(-\left(\mathrm{CH}_{3}\right)_{3}, \mathrm{NH}, \mathrm{CH}\right.$ and $\mathrm{CH}_{2}$ from polymer backbone), $\delta$ 3.10-3.90 ppm $\left(-\mathrm{CH}_{2}-\mathrm{NH}\right), \delta 6.00-7.2 \mathrm{ppm}$ (aromatic $\left.\mathrm{H}\right) ;{ }^{13} \mathrm{C}$ NMR: $\delta 29.4 \mathrm{ppm}$ $\left(-\left(\mathrm{CH}_{3}\right)_{3}\right), \delta 40.4 \mathrm{ppm}\left(\mathrm{CH}_{2}\right.$ from polymer backbone), $\delta 41.5-45.5 \mathrm{ppm}$ ( $\mathrm{CH}$ from polymer backbone), $\delta 47.0-47.3 \mathrm{ppm}\left(-\mathrm{CH}_{2}-\mathrm{NH}\right), \delta 50.8 \mathrm{ppm}\left(-\left(\mathrm{CH}_{3}\right)_{3}\right), \delta 124.0-129.0 \mathrm{ppm}($ aromatic $\mathrm{CH}) \delta 138.8,141.0$, $143.9,145.5$ ppm (Quart-aromatic C).

\subsection{Preparation of Samples for Antimicrobial Assay}

For each sample $125 \mathrm{mg}$ of purified polymer was dissolved in $3 \mathrm{~mL}$ ethanol under stirring. The solution was then cast in a petri dish without vents (polystyrene, $d=90 \mathrm{~mm}, h=14.2 \mathrm{~mm}, \mathrm{VWR}$, Langenfeld, Germany) and dried in a vacuum drying cabinet (Heraeus Vacutherm VT 6025, Thermo Scientific, Waltham, MA, USA) at $70{ }^{\circ} \mathrm{C}$ and $2 \mathrm{mbar}$ for $1 \mathrm{~h}$. 


\subsection{Antimicrobial Assessment}

The antimicrobial activity of purified poly(TBAMS) was tested according to the Antimicrobial products-Test for antimicrobial activity and efficacy (JIS Z 2801:2000). In each test three poly(TBAMS) samples and three blank petri dishes of the same size were used as reference. Staphylococcus aureus ssp. aureus (DSM No. 799) and Escherichia coli (DSM No. 1576) were applied as test organisms. The inoculum was prepared by transferring a frozen culture to $10 \mathrm{~mL}$ nutrient broth (Roth, Karlsruhe, Germany), afterwards the broth was incubated at $37{ }^{\circ} \mathrm{C}$ for $24 \mathrm{~h}$. In the beginning of each trial the inoculum was diluted in physiological saline solution with tryptone (Oxoid, Hampshire, UK) to a final concentration of $10^{5} \mathrm{cfu} / \mathrm{mL}$. Samples and references were inoculated with $0.4 \mathrm{~mL}$ of this solution. To prevent evaporation and to standardize the contact area, the inoculums were covered loosely by sterile PE films $(40 \times 40 \mathrm{~mm})$.

Three references were directly washed out with $10 \mathrm{~mL}$ soybean casein digest broth with lecithin polysorbat (Roth, Karlsruhe, Germany) to determine the starting concentration. The poly(TBAMS) samples and the remaining references were incubated at $35{ }^{\circ} \mathrm{C}$ and $90 \%$ humidity for $2 \mathrm{~h}$. Afterwards all samples were washed in a similar manner. Viable counts were determined by counting the colonies on plate count agar (Roth, Karlsruhe, Germany) which were incubated for $48 \mathrm{~h}$ at $37^{\circ} \mathrm{C}$.

The value of antimicrobial activity were calculated by subtracting the logarithmic value of viable counts on poly(TBAMS) from the logarithmic value of reference material after inoculation and incubation:

$$
\log _{10}-\text { Reduction }=\log _{10}\left(\frac{\operatorname{Ref}_{2 h}}{\operatorname{poly(TBAMS)_{2h}}}\right)
$$

whereas $\mathrm{cfu}_{(T \mathrm{x}, \text { reference })}=$ arithmetic mean of bacterial counts on reference material $2 \mathrm{~h}$ after inoculation, and $\mathrm{cfu}_{(T \mathrm{x}, \text { sample })}=$ arithmetic mean of bacterial counts on poly(TBAMS) material $2 \mathrm{~h}$ after inoculation.

According to the JIS Z 2801:2000, a material can be characterized as antimicrobial, if the calculated $\log _{10}$-reduction is $\geq 2.0$ after $24 \mathrm{~h}$ at $35^{\circ} \mathrm{C}$.

\subsection{Incubation of Poly(TBAMS) in Escherichia coli (DSM 1576) Culture for SEM Imaging}

In a petri dish containing the poly(TBAMS) film there was added $4.5 \mathrm{~mL}$ of an overnight culture of Escherichia coli in a complex medium (0.8 g microbiology broth (order No. 1.05443.0500, Merck), $0.84 \mathrm{~g} \mathrm{NaCl}$, and $0.1 \mathrm{~g}$ Trypton (Applichem, Darmstadt, Germany) and $100 \mathrm{~mL}$ using $\mathrm{H}_{2} \mathrm{O}, \mathrm{pH}$ 7.4). Blank petri dishes were used as reference. The petri dishes were incubated at room temperature for $12 \mathrm{~h}$. The bacteria suspension was transferred into a $15 \mathrm{~mL}$ centrifuge tube, the bacteria were fixed chemically using $2.5 \mathrm{~mL}$ of a aqueous $2 \%$ glutaraldehyde solution at $4{ }^{\circ} \mathrm{C}$ for $3 \mathrm{~h}$. After this the suspension was centrifuged for $10 \mathrm{~min}$ at $400 \mathrm{rpm}$ to harvest the bacteria. The supernatant was discarded and the bacteria were dehydrated for $15 \mathrm{~min}$ in a series of ethanol/water solutions of increasing ethanol content (30\%, $50 \%, 70 \%$ and 90\%). The last drying step was repeated two times using absolute ethanol. Ethanol was removed in a vacuum exsiccator at 500 mbar. The pellets were transferred to a SEM sample holder. Gold was deposited on the specimens with an ion spatter. For scanning electron microscopy a Leo 982 “Gemini” (Zeiss, Jena, Germany) microscope was used. 


\section{Conclusions}

The preparation and characterization of TBAMS and poly(TBAMS) was described in this study. The synthesis of TBAMS from commercially available starting materials was straightforward and high yielding. Polymerization to poly(TBAMS) generated a new intrinsic antimicrobial polymer with an acceptable glass transition temperature and good film building properties. Excellent antimicrobial action against $E$. coli and $S$. aureus was observed. First insights from the SEM experiments showed that the antimicrobial activity correlates with the disruption of the cell membranes. Further experiments elucidating the applicability of poly(TBAMS) as a antimicrobial additive in compounds are currently underway.

\section{Acknowledgments}

The authors wish to thank all co-workers who have contributed to the work. The work was funded by the Bundesministerium für Wirtschaft und Technologie (BMWi) in the InnoNet program contract number 16INO640 (Verbund-Nr. 01063175) (Smart Surf).

\section{Author Contributions}

Reinhard Lorenz and Martin Kreyenschmidt conceived and designed the experiments regarding synthetic work in collaboration with Björn Fischer, Katrin Kalbfleisch, Oliver Robers and Florian Brodkorb. Katrin Kalbfleisch and Björn Fischer performed experiments regarding synthetic work. Oliver Robers and Florian Brodkorb performed the experiments regarding characterization and purification of the monomers and polymers. Microbiology studies were designed by Judith Kreyenschmidt, Carina Braun and Sophia Dohlen. The log reduction of the polymers was determined by Carina Braun and Sophia Dohlen. Florian Brodkorb carried out the SEM imaging experiments and wrote the paper in close cooperation with all authors. All authors read and approved the final manuscript.

\section{Conflicts of Interest}

The authors declare no conflict of interest.

\section{References}

1. Camilloto, G.P.; Pires, A.C.S.; Soares, N.F.F.; Araujo, E.A.; Andrade, N.J.; Ferreira, S.O. Effect of active packaging incorporated with triclosan on bacteria adhesion. J. Food Sci. 2010, 75, E557-E564.

2. Ohe, P.C.; Schmitt-Jansen, M.; Slobodnik, J.; Brack, W. Triclosan-The forgotten priority substance? Environ. Sci. Pollut. Res. 2012, 19, 585-591.

3. Iconomopoulou, S.M.; Andreopoulou, A.K.; Soto, A.; Kallitsis, J.K.; Voyiatzis, G.A. Incorporation of low molecular weight biocides into polystyrene-divinyl benzene beads with controlled release characteristics. J. Control. Release 2005, 102, 223-233.

4. Sun, X.; Cao, Z.; Porteous, N.; Sun, Y. Amine, melamine, and amide $N$-halamines as antimicrobial additives for polymers. Ind. Eng. Chem. Res. 2010, 49, 11206-11213. 
5. Maillard, J.-Y.; Hartemann, P. Silver as an antimicrobial: Facts and gaps in knowledge. Crit. Rev. Microbiol. 2013, 39, 373-383.

6. Martinez-Abad, A. Silver- and Nanosilver-Based Plastic Technologies; John Wiley \& Sons, Inc.: Hoboken, NJ, USA, 2012; pp. 287-316.

7. Borkow, G. Using copper to fight microorganisms. Curr. Chem. Biol. 2012, 6, 93-103.

8. Pereyra, A.M.; Gonzalez, M.R.; Rodrigues, T.A.; Soares Luterbach, M.T.; Basaldella, E.I. Enhancement of biocorrosion resistance of epoxy coating by addition of $\mathrm{Ag} / \mathrm{Zn}$ exchanged a zeolite. Surf. Coat. Technol. 2015, 270, 284-289.

9. Munoz-Bonilla, A.; Fernandez-Garcia, M. The roadmap of antimicrobial polymeric materials in macromolecular nanotechnology. Eur. Polym. J. 2015, 65, 46-62.

10. Munoz-Bonilla, A.; Fernandez-Garcia, M. Polymeric materials with antimicrobial activity. Prog. Polym. Sci. 2012, 37, 281-339.

11. Gerba, C.P. Quaternary ammonium biocides: Efficacy in application. Appl. Environ. Microbiol. 2015, 81, 464-469.

12. Lundin, J.G.; Coneski, P.N.; Fulmer, P.A.; Wynne, J.H. Relationship between surface concentration of amphiphilic quaternary ammonium biocides in electrospun polymer fibers and biocidal activity. React. Funct. Polym. 2015, 77, 39-46.

13. Liu, F.; Chang, X.; Yang, F.; Dong, W.; Wang, F.; Zhao, C. Effects of biocides on biofilm at different substrate levels. Prog. Environ. Sci. Technol. 2011, 3, 632-636.

14. Oh, S.; Tandukar, M.; Pavlostathis, S.G.; Chain, P.S.G.; Konstantinidis, K.T. Microbial community adaptation to quaternary ammonium biocides as revealed by metagenomics. Environ. Microbiol. 2013, 15, 2850-2864.

15. Valle, A.S.S.; Marques, M.R.C.; Costa, L.C.; Santa Maria, L.C.; Palermo de Aguiar, A.; Mercon, F. Evaluation of bactericidal action of 2-vinylpyridine copolymers containing quaternary ammonium groups and their charge transfer complexes. Polim. Cienc. Tecnol. 2013, 23, 152-160.

16. Carmona-Ribeiro, A.M.; de Melo Carrasco, L.D. Cationic antimicrobial polymers and their assemblies. Int. J. Mol. Sci. 2013, 14, 9906-9946.

17. Xue, Y.; Xiao, H.; Zhang, Y. Antimicrobial polymeric materials with quaternary ammonium and phosphonium salts. Int. J. Mol. Sci. 2015, 16, 3626-3655.

18. Bansal, R.; Pathak, R.; Jha, D.; Kumar, P.; Gautam, H.K. Enhanced antimicrobial activity of amine-phosphonium (N-P) hybrid polymers against gram-negative and gram-positive bacteria. Int. J. Polym. Mater. Polym. Biomater. 2014, 64, 84-89.

19. Choi, S.Y.; Rodriguez, H.; Gunaratne, H.Q.N.; Puga, A.V.; Gilpin, D.; McGrath, S.; Vyle, J.S.; Tunney, M.M.; Rogers, R.D.; McNally, T. Dual functional ionic liquids as antimicrobials and plasticizers for medical grade pvcs. RSC Adv. 2014, 4, 8567-8581.

20. Chang, H.-I.; Yang, M.-S.; Liang, M. The synthesis, characterization and antibacterial activity of quaternized poly(2,6-dimethyl-1,4-phenylene oxide)s modified with ammonium and phosphonium salts. React. Funct. Polym. 2010, 70, 944-950.

21. Kratky, M.; Vinsova, J. Antimycobacterial activity of quaternary pyridinium salts and pyridinium N-oxides-Review. Curr. Pharm. Des. 2013, 19, 1343-1355.

22. Al-Sha'er, M.A. Evaluation of antimicrobial activities of synthesized pyridinium derivatives. Pharma Chem. 2014, 6, 261-291. 
23. Furdui, B.; Constantin, O.; Dinica, R.; Bahrim, G. Studies on antimicrobial activities of some new bis-pyridinium derivatives. Ann. Univ. Dunarea Jos Galati Fasc. VI 2011, 35, 66-74.

24. Inhester, M.; Ottersbach, P. Kosmetische formulierungen mit antimikrobiellen polymeren. WO2003068316 A1, 21 August 2003. (In German)

25. Kossmann, B.D.I.D.; Ottersbach, P.D.C.D. Antimikrobielle polymere als konservierungsstoffe für flüssige lebensmittel. DE10244489 A1, 25 March 2004. (In German)

26. Ottersbach, P.; Kossmann, B. Verfahren zum einsatz antimikrobieller polymere im bauten- und denkmalschutz. WO2002048070 A1, 20 June 2002. (In German)

27. Ottersbach, P.; Kossmann, B. Antimikrobielle fasern und gewebe. DE10122753 A1, 14 November 2002. (In German)

28. Paleari, A.G.; Marra, J.; Pero, A.C.; Rodriguez, L.S.; Ruvolo-Filho, A.; Compagnoni, M.A. Effect of incorporation of 2-tert-butylaminoethyl methacrylate on flexural strength of a denture base acrylic resin. J. Appl. Oral Sci. 2011, 19, 195-199.

29. Marra, J.; Paleari, A.G.; Rodriguez, L.S.; Leite, A.R.P.; Pero, A.C.; Compagnoni, M.A. Effect of an acrylic resin combined with an antimicrobial polymer on biofilm formation. J. Appl. Oral Sci. 2012, 20, 643-648.

30. Ottersbach, P.D.; Sosna, F.D. Verfahren zur mikrobiziden tiefenimprägnierung poröse materialien. EP1281490 A1, 5 February 2003.

31. Buranasompob, A. Kinetics of the inactivation of microorganisms by water insoluble polymers with antimicrobial activity. Ph. D. Thesis, Technische Universität Berlin, Berlin, Germany, 7 March 2005.

32. Kreyenschmidt, M.; Lorenz, R.; Fischer, B.; Kreyenschmidt, J.; Brodkorb, F.; Kalbfleisch, K.; Blang, T.; Geschwentner, A. Anti-microbial polymer. WO2014118339 A1, 7 August 2014.

33. Sakurai, K.; Maegawa, T.; Takahashi, T. Glass transition temperature of chitosan and miscibility of chitosan/poly( $n$-vinyl pyrrolidone) blends. Polymer 2000, 41, 7051-7056.

34. Dhawade, P.P.; Jagtap, R.N. Characterization of the glass transition temperature of chitosan and its oligomers by temperature modulated differential scanning calorimetry. Adv. Appl. Sci. Res. 2012, 3, 1372-1382.

35. Kittur, F.S.; Harish Prashanth, K.V.; Udaya Sankar, K.; Tharanathan, R.N. Characterization of chitin, chitosan and their carboxymethyl derivatives by differential scanning calorimetry. Carbohydr. Polym. 2002, 49, 185-193.

36. Jeong, J.-H.; Byoun, Y.-S.; Lee, Y.-S. Poly(styrene-alt-maleic anhydride)-4-aminophenol conjugate: Synthesis and antibacterial activity. React. Funct. Polym. 2002, 50, 257-263.

37. Dizman, B.; Elasri, M.O.; Mathias, L.J. Synthesis and characterization of antibacterial and temperature responsive methacrylamide polymers. Macromolecules 2006, 39, 5738-5746.

38. Lenoir, S.; Pagnoulle, C.; Galleni, M.; Compere, P.; Jerome, R.; Detrembleur, C. Polyolefin matrixes with permanent antibacterial activity: Preparation, antibacterial activity, and action mode of the active species. Biomacromolecules 2006, 7, 2291-2296.

39. Endo, Y.; Tani, T.; Kodama, M. Antimicrobial activity of tertiary amine covalently bonded to a polystyrene fiber. Appl. Environ. Microbiol. 1987, 53, 2050-2055. 
40. Park, S.-C.; Nam, J.-P.; Kim, J.-H.; Kim, Y.-M.; Nah, J.-W.; Jang, M.-K. Antimicrobial action of water-soluble $\beta$-chitosan against clinical multi-drug resistant bacteria. Int. J. Mol. Sci. 2015, 16, 7995-8007.

(C) 2015 by the authors; licensee MDPI, Basel, Switzerland. This article is an open access article distributed under the terms and conditions of the Creative Commons Attribution license (http://creativecommons.org/licenses/by/4.0/). 